Running head: Procrastination and the future

\title{
Procrastination, consideration of future consequences, and episodic future thinking
}

Marie My Lien Rebetez ${ }^{\mathrm{a}, \mathrm{b}}$, Catherine Barsics ${ }^{\mathrm{a}, \mathrm{b}}$, Lucien Rochat ${ }^{\mathrm{a}, \mathrm{b}}$, Arnaud D’Argembeau ${ }^{\mathrm{b}, \mathrm{c}, \mathrm{d}}$, and Martial Van der Linden ${ }^{\text {a,b,c }}$ 


\begin{abstract}
Despite the intrinsic temporal nature of procrastination, little research has examined the link between this form of self-regulatory failure and the consideration of future consequences, and no study has addressed the link between procrastination and episodic future thinking. The aim of the present study was to explore these relationships. Participants were asked to project themselves into possible future events and to rate the amount of sensory-perceptual details and autonoetic consciousness associated with their representations. They were also asked to complete questionnaires that assessed procrastination, the consideration of future consequences, and negative affect. Results showed that both the consideration of future consequences and episodic future thinking were associated with procrastination, and in particular with procrastination-related decision making abilities and procrastination-related motivational dispositions, respectively.
\end{abstract}

Keywords: Procrastination; time perspective; consideration of future consequences; prospection; episodic future thinking. 


\section{Introduction}

Procrastination - voluntarily delaying an intended and necessary and/or [personally] important activity, despite expecting potential negative consequences that outweigh the positive consequences of the delay (Klingsieck, 2013) - is a widespread phenomenon that has been established as a stable trait and conceptualized as a self-regulatory failure (for a review, see Klingsieck, 2013; Rozental \& Carlbring, 2014; Schouwenburg, Lay, Pychyl, \& Ferrari, 2004; Steel, 2007). More specifically, recent theory suggests that procrastination is a form of temporal self-regulation failure, representative of high impulsiveness (Gustavson, Miyake, Hewitt, \& Friedman, 2014) and reflecting a primacy of present self over the needs of the future self (Sirois $\&$ Pychyl, 2013). This failure to regulate the self over time may lead procrastinators to experience more negative emotions, such as shame and guilt (Fee \& Tangney, 2000; Flett, Stainton, Hewitt, Sherry, \& Lay, 2012), and generally to experience less well-being (Sirois \& Tosti, 2012).

Given its intrinsic temporal nature, procrastination has been associated with individual differences in time perspective (dispositional style toward being past-, future-, or presentoriented, which reflects processes involving how the flow of experiences are assigned to temporal frames that help to give order, coherence and meaning to these events; Zimbardo \& Boyd, 1999). In particular, procrastination has been consistently related to a low level of future time perspective (for a review, see Díaz-Morales \& Ferrari, 2015; Sirois, 2014). Procrastinators are thus less likely to use a future time orientation to guide their decisions and actions (Díaz-Morales, Ferrari, \& Cohen, 2008; Ferrari \& Díaz-Morales, 2007; Gupta, Hershey, \& Gaur, 2012; Jackson, Fritch, Nagasaka, \& Pope, 2003). Moreover, Sirois (2004) looked at the link between procrastination and a specific form of future time orientation, namely, the consideration of future consequences (the extent to which people consider future versus immediate consequences of potential behaviors; Strathman, Gleicher, Boninger, \& Edwards, 1994). Procrastinators were less likely to consider the potential future outcomes of their current behavior. These data suggest that procrastination involves a decisional conflict between the immediate and delayed consequences of one's behavior.

However, envisioning what might happen in the future requires not only to orient oneself towards a future time period, but also to construct a detailed representation of events that could occur. This distinction is important because recent evidence suggests that temporal orientation and event construction are dissociable processes. For example, Kwan et al. (2012) demonstrated that a person with episodic amnesia, who was unable to imagine possible future events, still systematically discounted the value of future rewards (which requires future orientation but not event construction). Nevertheless, the ability to imagine possible future events may interact with future-oriented decision making (and/or temporal orientation) to support the representation of what might happen in the future (Szpunar, Spreng, \& Schacter, 2014). This ability to imagine possible future events - often termed "episodic future thinking" - implies the association and flexible recombination of elements stored in episodic memory, such as details about particular locations, persons, and objects (Schacter \& Addis, 2007; Suddendorf \& Corballis, 2007). These details are represented as simulations in modality-specific systems (Barsalou, 2008) and give rise to mental images subjectively perceived as more or less vivid (D'Argembeau \& Van der Linden, 2006). In addition to these sensory-perceptual qualities, episodic future thoughts are accompanied by "cognitive feelings" (Conway, 2009), including autonoetic consciousness (i.e., the subjective sense of being brought forward in time to pre-experience an event; Tulving, 2002; Wheeler, Stuss, \& Tulving, 1997). Such cognitive feelings are of central importance, as they influence one's beliefs about what might happen in the future, which in turn influence one's decisions and actions (Libby, Shaeffer, Eibach, \& Slemmer, 2007). Overall, the ability to imagine plausible 
future events is deemed to have a strong adaptive value in daily life, in particular, by supporting goal-directed behaviors. For example, mental simulation of future events facilitates the link between goals and actions by enhancing the subjective likelihood and/or value of a goal (which increases motivation) and by detailing the steps necessary to attain a goal (Gregory, Cialdini, \& Carpenter, 1982; Karniol \& Ross, 1996; Raffard, Esposito, Boulenger, \& Van der Linden, 2013; Taylor, \& Schneider, 1989; Taylor, Pham, Rivkin, \& Armor, 1998).

Episodic future thinking is thus critical for anticipating future needs and states that allow for better planning and understanding of the consequences of one's behaviors. In this respect, difficulties in imagining the future may be linked to procrastination - conceptualized as a selfregulatory failure (see Steel, 2007) - but no study has yet, to our knowledge, examined this link. Furthermore, while the link between procrastination and general orientation towards the future has been examined several times (see Díaz-Morales \& Ferrari, 2015; Sirois, 2014), the link with a more specific form of future time orientation (i.e., the consideration of future consequences) has received little attention. The way in which the consideration of future consequences and episodic future thinking contribute to procrastination could be related to two factors that have been recently shown to underlie the French version of the Pure Procrastination Scale (Rebetez, Rochat, Gay, \& Van der Linden, 2014): "Voluntary delay," which refers to the notion of irrationally and/or voluntarily putting off things or decisions, and "Observed delay," which relates to noticing that one is running out of time, not getting things done on time, or not being very good at meeting deadlines. These two factors depended on a higher-order construct of procrastination and were differentially associated with measures of impulsivity. Indeed, Voluntary delay was related to lack of premeditation dimension (the tendency to not take into account the consequences of an act before engaging in that act; Whiteside \& Lynam, 2001), suggesting a preference to act on the spur of the moment, disregarding the long-term consequences of delaying. On the other hand, Observed delay was related to sensation seeking (the tendency to enjoy and pursue new/exciting activities; Whiteside \& Lynam, 2001) and therefore might be a manifestation of stimulation seeking (e.g., getting the "rush" from completing the task close to the deadline; Ferrari, 1992).

In this context, the objective of the present study was to explore the relationships between procrastination (both Voluntary and Observed delay), the consideration of future consequences, and episodic future thinking. Participants were thus asked to project themselves into possible future events and to rate the amount of sensory-perceptual details and the level of autonoetic consciousness associated with their representations. They further completed questionnaires assessing procrastination, the consideration of future consequences, and negative affect (included as a control variable given its close connection with procrastination; see Fee \& Tangney, 2000; Flett et al., 2012; Sirois \& Pychyl, 2013).

The following predictions were tested. First, we predicted that procrastination (and more specifically Voluntary delay, i.e., the procrastination factor associated with lack of premeditation) would relate to a low level of consideration of future consequences. Second, regarding the relationships between procrastination and episodic future thinking, we predicted that procrastination (both Voluntary delay and Observed delay) would relate to lower episodic future thinking abilities (as measured by the amount of sensory-perceptual qualities and the level of autonoetic consciousness when imagining future events). Indeed, mental simulation of future events facilitates the link between goals and actions (see Karniol \& Ross, 1996; Raffard et al., 2013; Taylor et al., 1998); by contrast, poor representations of future events may contribute to the intention-action gap that characterizes procrastination. 


\subsection{Participants and procedure}

\section{Method}

Participants were 103 undergraduate students (63 females, 40 males), who were recruited through advertisement and personal contacts; they received no compensation for their participation. The mean age of the sample was 23.17 years $(S D=3.94$, range $=18-35)$. The inclusion criterion was being a fluent speaker of French. Exclusion criteria were a reported history of neurological disorder or psychiatric condition involving medication usage. Participants were tested individually in a quiet environment. They signed an informed consent form before completing the task and questionnaires assessing episodic future thinking, procrastination, the consideration of future consequences, and negative affect. The order of presentation of these measures was counterbalanced across participants. The study was approved by the Ethics Committee of the University of Geneva.

\subsection{Measures}

\subsubsection{Episodic future thinking}

Episodic future thinking (EFT) was assessed with an adaptation of a procedure developed by D'Argembeau and Van der Linden (2012). Participants were instructed to imagine a series of future events, from any future time period, but that might plausibly happen to them. These events had to be new (i.e., no recount of an actual memory) and specific (i.e., an event that happens in a specific place, at a specific time, and that lasts no more than a day). Examples were provided to illustrate the kinds of events that would or would not be considered specific. Participants were then asked to imagine five plausible, new, and specific events in response to cue words (i.e., an event that the cues made them think of), in as much detail as possible, using all their senses, as if they were physically present. Each cue word, whose order was counterbalanced, was provided orally and referred to familiar persons and broad categories of experiences (i.e. family, work, friend, party, and trip) that could especially be associated with a variety of specific future events (see D'Argembeau \& Van der Linden, 2012).

After having imagined and described each event, participants had to assess the phenomenological characteristics of their future thoughts, using a series of 7-point rating scales that were adapted from previous work on episodic future thinking (D'Argembeau \& Van der Linden, 2004, 2006, 2012). Five items assessed the sensory-perceptual qualities of the future imagined event: overall vividness $(1=$ very vague, $7=$ very vivid $)$, amount of visual details $(1=$ not at all, $7=\mathrm{a}$ lot $)$, amount of other sensory details $(1=$ not at all, $7=\mathrm{a}$ lot $)$, clarity of location $(1$ = very vague, $7=$ very clear $)$, and clarity of persons and objects $(1=$ very vague, $7=$ very clear $)$. An index of sensory-perceptual qualities (EFT-Sensory-perceptual qualities) was computed by averaging responses to these items. Two other items, which were regrouped into an index of autonoetic consciousness (EFT-Autonoetic consciousness), assessed the subjective sense of being brought forward in time $(1=$ not at all, $7=\mathrm{a}$ lot $)$ and the feeling of pre-experiencing the event $(1$ $=$ not at all, $7=\mathrm{a}$ lot $)$.

\subsubsection{Procrastination}

Procrastination was measured with the French adaptation of the Pure Procrastination Scale (PPS; Rebetez et al., 2014; original version, Steel, 2010), an 11-item_questionnaire which allows to distinguish between Voluntary delay ( 8 items, e.g., "I am continually saying I'll do it tomorrow") and Observed delay (3 items, e.g., "I find myself running out of time"). In the French validation of the PPS (Rebetez et al., 2014), exploratory and confirmatory analyses revealed that the scale was composed of two factors (PPS-Voluntary delay; PPS-Observed delay) depending on a common, higher order construct of procrastination. PPS-Voluntary delay and PPS-Observed delay had acceptable to good internal reliability. In addition, good test-retest reliability was 
found, and external validity was supported by specific relationships with measures of personality traits, impulsivity, and subjective well-being. In particular, PPS-Voluntary delay was specifically related to lack of premeditation dimension of impulsivity and PPS-Observed delay to sensation seeking dimension of impulsivity. Higher scores indicate a higher tendency to procrastinate.

\subsubsection{Consideration of future consequences}

The consideration of future consequences was assessed with the French adaptation of the Consideration of Future Consequences Scale (CFC; Demarque, Apostolidis, Chagnard, \& Dany, 2010; original version, Strathman et al., 1994), a 7-item questionnaire (e.g., "Often I engage in a particular behavior in order to achieve outcomes that may not result for many years") for which a higher score indicates a higher level of consideration of future consequences. Exploratory and confirmatory factor analyses of the French adaptation of the CFC indicated a one-factor model with acceptable internal reliability score; good test-retest reliability was found, and convergent, divergent and predictive validity was satisfactory (Demarque et al., 2010).

\subsubsection{Negative affect}

Negative affect was assessed with the French version of the Positive Affect Negative Affect Schedule (PANAS; Gaudreau, Sanchez, \& Blondin, 2006; original version, Watson, Clark, \& Tellegen, 1988). This 20 -item scale evaluates the tendency to experience positive (10 items; e.g., "Happy") or negative affect (10 items; e.g., "Upset"). In the initial preliminary validation study of the PANAS (Watson et al., 1988), factorial validity, test-retest reliability, convergent and divergent validity for the positive affect and the negative affect scales have been demonstrated._For this study, only negative affect (PANAS-) was considered. A higher score indicates a higher tendency to experience negative affect.

\section{Results}

Prior to data analyses, skewness and kurtosis were examined for all scales. For each variable, they ranged from - -25 to .52 and -.85 to .94 , respectively, indicating no strong deviation from normality, considering that absolute values greater than 3 for skewness and greater than 20 for kurtosis are judged to be extreme (Weston \& Gore, 2006). Means, standard deviations, internal consistency reliability coefficients, and ranges of the measures are reported in Table 1. The Cronbach's $\alpha$ ranged from .80 to .92 , indicating acceptable to excellent reliability (a value above .70 is acceptable, above .80, good, and above .90, excellent; George \& Mallery, 2005).

As Table 2 shows, Pearson's correlations were computed between procrastination scores (PPS-Voluntary delay, PPS-Observed delay), negative affect (PANAS-), the consideration of future consequences (CFC), the amount of sensory-perceptual qualities when imagining future events (EFT-Sensory-perceptual qualities), and the level of autonoetic consciousness accompanying episodic future thoughts (EFT-Autonoetic consciousness). PPS-Voluntary delay and PPS-Observed delay were significantly positively related to the PANAS- and negatively related to the CFC and the EFT-Sensory-perceptual qualities. No relationship was found with the EFT-Autonoetic consciousness.

Regression analyses (Table 3) were then performed in order to examine the specific contribution of the CFC, the EFT-Sensory perceptual qualities, and the EFT-Autonoetic consciousness to each PPS score. We thus computed multiple linear regressions with PPSVoluntary delay and PPS-Observed delay as the criterion and with the PANAS-, the CFC, the EFT-Sensory-perceptual qualities, and the EFT-Autonoetic consciousness as independent variables. Note that the PANAS- was included as a predictor in order to control for negative affect. When performing the regression analyses with the EFT-Sensory-perceptual qualities and the EFT-Autonoetic consciousness, particular attention was paid to signs of multicollinearity 
Running head: Procrastination and the future

because of the strong correlation between these two variables $(r=.58, p<.001)$. We therefore used the variance inflation factor (VIF), which shows the amount by which the variance of the coefficient estimate is being inflated by multicollinearity, and the tolerance score. VIF values over 2.50 and tolerance below .40 are considered problematic for multicollinearity (Allison, 1999). Accordingly, no multicollinearity was emphasized (all VIFs $<2.50$ and tolerance indices > .40). In addition, exploration of the residuals suggested that they were normally distributed. Results revealed that PPS-Voluntary delay was best predicted by the CFC, followed by the PANAS- and the EFT-Sensory-perceptual qualities. PPS-Observed delay was best predicted by the EFT-Sensory-perceptual qualities, followed by the CFC and then the PANAS-. 
Running head: Procrastination and the future

Table 1

Means, standard deviations, Cronbach's $\alpha$, and ranges of the measures

\begin{tabular}{lcccc}
\hline & $M$ & $S D$ & $\alpha$ & Range \\
\hline PPS-Voluntary delay & & & & 0.92 \\
PPS-Observed delay & 3.22 & 0.87 & 0.84 & $1-5$ \\
PANAS- & 2.57 & 1.01 & 0.81 & $1-5$ \\
CFC & 2.09 & 0.59 & 0.80 & $1-5$ \\
EFT-Sensory-perceptual qualities & 3.46 & 0.74 & 0.82 & $1-5$ \\
EFT-Autonoetic consciousness & 4.74 & 0.76 & 0.85 & $1-5$
\end{tabular}

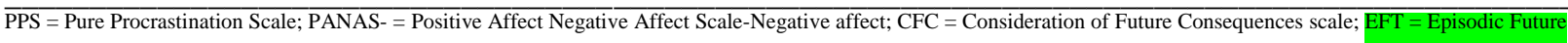

Thinking task

Table 2

Pearson's correlations between PPS scores and the other measures

\begin{tabular}{lll}
\hline & & \\
& PPS-Voluntary delay & PPS-Observed delay \\
\hline PANAS- & $.20 *$ & $.20^{*}$ \\
CFC & $-.37 * * *$ & $-.22^{*}$ \\
EFT-Sensory-perceptual qualities & $-.25 *$ & $-.24 *$ \\
EFT-Autonoetic consciousness & -.12 & -.05 \\
& & \\
PPS = Pure Procrastination Scale; PANAS- = Positive Affect Negative Affect Scale-Negative affect; CFC = Consideration of Future Consequences scale; EFT = Episodic Future \\
Thinking task \\
${ }^{*}$ Significant at $p<.05, * * * p<.001$ &
\end{tabular}

Table 3

PPS scores regressed on the CFC, the EFT-Sensory-perceptual qualities, and the EFT-Autonoetic consciousness, while controlling for the PANAS-

\begin{tabular}{|c|c|c|c|c|c|c|c|c|}
\hline \multirow[b]{2}{*}{ Predictor } & \multicolumn{3}{|c|}{ PPS-Voluntary delay } & \multicolumn{5}{|c|}{ PPS-Observed delay } \\
\hline & $\mathrm{B}$ & SE & $\beta$ & $t$ & B & $\mathrm{SE}$ & $\beta$ & $t$ \\
\hline Constant & 5.11 & .65 & & 7.87 & 4.12 & .79 & & 5.22 \\
\hline PANAS- & .30 & .13 & .20 & $2.22 *$ & .32 & .16 & .19 & $2.00 *$ \\
\hline $\mathrm{CFC}$ & -.41 & .11 & -.35 & $-3.93 * * *$ & -.28 & .13 & -.21 & $-2.18 *$ \\
\hline EFT-Sensory-perceptual qualities & -.26 & .13 & -.23 & $-2.07 *$ & -.39 & .15 & -.29 & $-2.52 *$ \\
\hline EFT-Autonoetic consciousness & .04 & .09 & .05 & .43 & .13 & .11 & .14 & 1.19 \\
\hline $\operatorname{adj} R^{2}$ & .19 & & & & .11 & & & \\
\hline
\end{tabular}




\section{Discussion}

Despite the intrinsic temporal nature of procrastination, little research has examined the link between this phenomenon and specific forms of future time orientation such as the consideration of future consequences, and no study has addressed the link between procrastination and episodic future thinking. Therefore, the aim of the current study was to explore the relationships between two distinct (although related) dimensions of procrastination (i.e., Voluntary delay and Observed delay), the consideration of future consequences, and episodic future thinking (as measured by the amount of sensory-perceptual qualities and the level of autonoetic consciousness associated with the imagination of future events).

Overall, our results indicate that both the level of consideration of future consequences and the amount of sensory-perceptual qualities when imagining future events (i.e., overall vividness; amount of visual and other sensory details; clarity of location, persons, and objects) contribute to procrastination. These relationships are specific and cannot be ascribed to a general tendency to experience negative affect, as they remained significant even when negative affect was controlled for. These results corroborate Sirois' study (2004) showing that procrastinators were less likely to consider the potential future outcomes of their current behavior. The results also suggest that procrastination is related to difficulties in simulating future events, as reflected by poor mental representations of imagined events (i.e., less vivid, with less details concerning places, people, and objects).

More specifically, the level of consideration of future consequences was the best predictor of Voluntary delay (i.e., the procrastination factor related to lack of premeditation). Lack of premeditation (i.e., the tendency to not take into account the consequences of an act before engaging in that act) is one of the dimensions of impulsivity (see Whiteside \& Lynam, 2001) that is considered to be underlain by decision-making abilities (see Bechara \& Van der Linden, 2005). Interestingly, Joireman, Sprott, and Spangenberg (2005) demonstrated that people with a low level of consideration of future consequences were more likely to discount the value of future rewards (one aspect of decision making) and to engage in impulsive behaviors. Tentatively, the decision-making process of postponing (or not postponing) actions/decisions raises a dilemma between short- versus long-term consequences of delaying, and the way people deal with such a temporal dilemma is likely to be shaped by the extent to which they are concerned with the immediate versus delayed consequences of their behaviors. If the level of consideration of future consequences is low, little weight is given to the long-term consequences of delaying during the decision-making process between short- and long-term goals, which results in the report of actions/decisions.

The amount of sensory-perceptual qualities when imagining future events was the best predictor of Observed delay (i.e., the procrastination factor related to sensation seeking). As sensation seeking (i.e., the tendency to enjoy and pursue new/exciting activities) is considered to depend on motivational dispositions (e.g., overactive appetitive motivational system and/or underactive aversive system; Lang, Shin, \& Lee, 2005), Observed delay reflects, at least in part, motivational aspects of delaying. Thus, the ability to imagine plausible future events in a specific manner, which is recognized to increase motivation by enhancing the subjective likelihood and/or value of a goal (see Karniol \& Ross, 1996; Raffard et al., 2013; Taylor et al., 1998), may play a role in the motivational aspects of delaying. It might be suggested that imagining a desired outcome with many details constitutes a source of motivation for achieving this imagined state; by contrast, poor mental representation of the outcome, in particular when the motivation to achieve this outcome is initially low, may lead to seek stimulation otherwise (e.g., by delaying the task). 
Running head: Procrastination and the future

Finally, the absence of any relationship between autonoetic consciousness accompanying episodic future thoughts (i.e., the subjective sense of being brought forward in time and the feeling of pre-experiencing the event) and procrastination, although sensory-perceptual qualities were related to procrastination, needs discussion. One possible explanation for these findings might lie in the fact that autonoetic consciousness, even though it depends on sensory perceptualqualities, also depends on the relevance of imagined future events with respect to personal goals and values (D'Argembeau \& Van der Linden, 2012). Accordingly, the relationship between autonoetic consciousness and procrastination might be modulated by the personal importance attributed to the events, irrespective of the amount of sensory-perceptual qualities.

Our study thus constitutes a first characterization of the links between procrastination and episodic future thinking. As the size of our sample was relatively small, future research should examine these links in a larger number of participants. A further step could consist in examining whether and how procrastination is particularly related to the capacity to imagine goal-related events. To examine this issue, one may combine future thinking tasks with procedures designed to elicit, rate and/or compare personal goals (e.g., Personal Project Analysis; Little, 1983). Further studies are also needed to examine how episodic future thinking and future time orientation interact with each other to support the representation of what might happen in the future, and ultimately, to determine whether the way someone imagines a future outcome and the course of action required to attain it determines the likelihood that a goal will be actually achieved. Finally, a recent study (Blouin-Hudon \& Pychyl, 2015) indicating that procrastination is linked to difficulties in the ability to form vivid mental images raises the question of whether procrastinators present particular difficulties with episodic future thinking or display a more general deficit in constructing complex mental images (irrespective of whether or not these images refer to the future). 
Running head: Procrastination and the future

\section{Acknowledgements}

We thank Alexandra Bugnon, Lena Magnenat, and Sascha Zuber for their contributions to data collection.

This research was supported by the National Center of Competence in Research (NCCR) Affective Sciences financed by the Swiss National Science Foundation ( $\left.{ }^{\circ} 51 N F 40-104897\right)$ and hosted by the University of Geneva. 
Running head: Procrastination and the future

\section{References}

Allison, P. D. (1999). Logistic regression using the SAS system: Theory and application. Cary, NC: SAS Institute.

Barsalou, L. W. (2008). Grounded cognition. Annual Review Psychology, 59, 617-645.

Bechara, A., \& Van der Linden, M. (2005). Decision-making and impulse control after frontal lobe injuries. Current Opinion in Neurology, 18, 734-739.

Blouin-Hudon, E. - M. C., \& Pychyl, T. A. (2015). Experiencing the temporally extended self: Initial support for the role of affective states, vivid mental imagery, and future selfcontinuity in the prediction of academic procrastination. Personality and Individual Differences, 86, 50-56.

Conway, M. A. (2009). Autobiographical memory and consciousness. In W. P. Banks (Eds.), Encyclopedia of consciousness (pp. 77-82). Oxford, United Kingdom: Academic Press.

D'Argembeau, A., \& Van der Linden, M. (2004). Phenomenal characteristics associated with projecting oneself back into the past and forward into the future: Influence of valence and temporal distance. Consciousness and Cognition, 13, 844-858.

D’Argembeau, A., \& Van der Linden, M. (2006). Individual differences in the phenomenology of mental time travel: The effect of vivid visual imagery and emotion regulation strategies. Consciousness and Cognition, 15, 342-350.

D’Argembeau, A., \& Van der Linden, M. (2012). Predicting the phenomenology of episodic future thoughts. Consciousness and Cognition, 21, 1198-1206.

Demarque, C., Apostolidis, T., Chagnard, A., \& Dany, L. (2010). Adaptation et validation française de l'échelle de perspective temporelle « consideration of future consequences » (CFC). Bulletin de Psychologie, 509, 351-360.

Díaz-Morales, J. F., \& Ferrari, J. R. (2015). More time to procrastinators: The role of time perspective. In M. Stolarski, N. Fieulaine, \& W. van Beek (Eds.), Time perspective theory; Review, research and application: Essays in honor of Philip G. Zimbardo (pp. 305-321). Cham, Switzerland: Springer.

Díaz-Morales, J. F., Ferrari, J. R., \& Cohen, J. R. (2008). Indecision and avoidant procrastination: The role of morningness-eveningness and time perspective in chronic delay lifestyles. The Journal of General Psychology, 135, 228-240.

Fee, R. L., \& Tangney, J. P. (2000). Procrastination: A means of avoiding shame or guilt? Journal of Social Behavior and Personality, 15, 167-184.

Ferrari, J. R. (1992). Psychometric validation of two procrastination inventories for adults: Arousal and avoidance measures. Journal of Psychopathology and Behavioral Assessment, 14, 97-110.

Ferrari, J. R., \& Díaz-Morales, J. F. (2007). Procrastination: Different time orientations reflect different motives. Journal of Research in Personality, 41, 707-714.

Flett, G. L., Stainton, M., Hewitt, P. L., Sherry, S. B., \& Lay, C. (2012). Procrastination automatic thoughts as a personality construct: An analysis of the procrastinatory cognitions inventory. Journal of Rational-Emotive and Cognitive-Behavior Therapy, 30, 223-236.

Gaudreau, P., Sanchez, X., \& Blondin, J.-P. (2006). Positive and negative affective states in a performance-related setting: Testing the factorial structure of the PANAS across two samples of French-Canadian participants. European Journal of Psychological Assessment, 22, 240-249.

George, D., \& Mallery, P. (2005). SPSS for Windows step by step: A simple guide and reference 12.0 update $\left(5^{\text {th }} \mathrm{ed}\right.$.). Boston, MA: Pearson Education. 
Running head: Procrastination and the future

Gregory, W. L. (1982), Cialdini, R. B., \& Carpenter, K. M. Self-relevant scenarios as mediators of likelihood estimates and compliance: Does imagining make it so? Journal of Personality and Social Psychology, 42, 89-99.

Gupta, R., Hershey, D. A., \& Gaur, J. (2012). Time perspective and procrastination in the workplace: An empirical investigation. Current Psychology, 31, 195-211.

Gustavson, D. E., Miyake, A., Hewitt, J. K., \& Friedman, N. P. (2014). Genetic relations among procrastination, impulsivity, and goal-management ability: Implications for the evolutionary origin of procrastination. Psychological Science, 25, 1178-1188.

Jackson, T., Fritch, A., Nagasaka, T., \& Pope, L. (2003). Procrastination and perceptions of past, present, and future. Individual Differences Research, 1, 17-28.

Joireman, J., Sprott, D. E., \& Spangenberg, E. R. (2005). Fiscal responsibility and the consideration of future consequences. Personality and Individual Differences, 39, 11591168.

Karniol, R., \& Ross, M. (1996). The motivational impact of temporal focus: Thinking about the future and the past. Annual Review of Psychology, 47, 593-620.

Klingsieck, K. B. (2013). Procrastination: When good things don't come to those who wait. European Psychologist, 18, 24-34.

Kwan, D., Craver, C. F., Green, L., Myerson, J., Boyer, P., \& Rosenbaum, R. S. (2012). Future decision-making without episodic mental time travel. Hippocampus, 22, 1215-1219.

Lang, A., Shin, M., \& Lee, S. (2005). Sensation seeking, motivation, and substance use: A dual system approach. Media Psychology, 7, 1-29.

Libby, L. K., Shaeffer, E. M., Eibach, R. P., \& Slemmer, J. A. (2007). Picture yourself at the polls: Visual perspective in mental imagery affects self-perception and behavior. Psychological Science, 18, 199-203.

Little, B. R. (1983). Personal projects: A rational and method for investigation. Environment and Behavior, 15, 273-309.

Raffard, S., Esposito, F., Boulenger, J.-P., \& Van der Linden, M. (2013). Impaired ability to imagine future pleasant events is associated with apathy in schizophrenia. Psychiatry Research, 209, 393-400.

Rebetez, M. M. L., Rochat, L., Gay, P., \& Van der Linden, M. (2014). Validation of a French version of the Pure Procrastination Scale (PPS). Comprehensive Psychiatry, 55, 14421447.

Rozental, A., \& Carlbring, P. (2014). Understanding and treating procrastination: A Review of a common self-regulatory failure. Psychology, 5, 1488-1502.

Schacter, D. L., \& Addis, D. R. (2007). The cognitive neuroscience of constructive memory: Remembering the past and imagining the future. Philosophical Transactions of the Royal Society of London B: Biological Sciences, 362, 773-786.

Schouwenburg, H. C., Lay, C. H., Pychyl, T. A., \& Ferrari, J. R. (2004). Counselling the procrastinator in academic settings. Washington, DC: American Psychological Association.

Sirois, F. M. (2004). Procrastination and intentions to perform health behaviors: The role of selfefficacy and the consideration of future consequences. Personality and Individual Differences, 37, 115-128.

Sirois, F. M. (2014). Out of sight, out of time? A meta-analytic investigation of procrastination and time perspective. European Journal of Personality, 28, 511-520. 
Running head: Procrastination and the future

Sirois, F. M., \& Pychyl, T. (2013). Procrastination and the priority of short-term mood regulation: Consequences for future self. Social and Personality Psychology Compass, 7 , 115-127.

Sirois, F. M., \& Tosti, N. (2012). Lost in the moment? An investigation of procrastination, mindfulness, and wellbeing. Journal of Rational-Emotive and Cognitive-Behavior Therapy, 30, 237-248.

Steel, P. (2007). The nature of procrastination: A meta-analytic and theoretical review of quintessential self-regulatory failure. Psychological Bulletin, 133, 65-94.

Steel, P. (2010). Arousal, avoidant and decisional procrastinators: Do they exist? Personality and Individual Differences, 48, 926-934.

Strathman, A., Gleicher, F., Boninger, D. S., \& Edwards, C. S. (1994). The consideration of future consequences: Weighing immediate and distant outcomes of behavior. Journal of Personality and Social Psychology, 66, 742-752.

Suddendorf, T., \& Corballis, M. C. (2007). The evolution of foresight: What is mental time travel, and is it unique to humans? Behavioral and Brain Sciences, 30, 299-351.

Szpunar, K. K., Spreng, R. N., \& Schacter, D. L. (2014). A taxonomy of prospection: Introducing an organizational framework of future-oriented cognition. Proceedings of the National Academy of Sciences USA, 111, 18414-18421.

Taylor, S. E., \& Schneider, S. K. (1989). Coping and the simulation of events. Social Cognition, 7, 174-194.

Taylor, S. E., Pham, L. B., Rivkin, I. D., \& Armor, D. A. (1998). Harnessing the imagination: Mental simulation, self-regulation, and coping. American Psychologist, 53, 429-439.

Tulving, E. (2002). Episodic memory: From mind to brain. Annual Review of Psychology, 53, 125.

Watson, D., Clark, L. A., \& Tellegen, A. (1988). Development and validation of brief measures of positive and negative affect: The PANAS scales. Journal of Personality and Social Psychology, 54, 1063-1070.

Weston, R., \& Gore, P. A. (2006). A brief guide to structural equation modeling. The Counseling Psychologist, 34, 719-751.

Wheeler, M. A., Stuss, D. T., \& Tulving, E. (1997). Toward a theory of episodic memory: The frontal lobes and autonoetic consciousness. Psychological Bulletin, 121, 331-354.

Whiteside, S. P., \& Lynam, D. R. (2001). The five factor model and impulsivity: Using a structural model of personality to understand impulsivity. Personality and Individual Differences, 30, 669-689.

Zimbardo, P. G., \& Boyd, J. N. (1999). Putting time in perspective: A valid, reliable, individualdifferences metric. Journal of Personality and Social Psychology, 77, 1271-1288. 